UMIACS TR 90-95

July 1990

CS TR 2504

\title{
On a Direct Method for the Solution of Nearly Uncoupled Markov Chains \\ G. W. STEWART* \\ G. $\mathrm{ZHANG}^{\dagger}$
}

\begin{abstract}
This note is concerned with the accuracy of the solution of nearly uncoupled Markov chains by a direct method based on the LU decomposition. It is shown that plain Gaussian elimination may fail in the presence of rounding errors. A modification of Gaussian elimination with diagonal pivoting as well as corrections of small pivots by sums of off-diagonal elements in the pivoting columns is proposed and analyzed. It is shown that the accuracy of the solution is affected by two condition numbers associate with the aggregate and the coupling respectively.
\end{abstract}

*Department of Computer Science and Institute for Advanced Computer Studies, University of Maryland, College Park, MD 20742. This work was supported in part by the Air Force Office of Sponsored Research under Contract AFOSR-87-0188.

${ }^{\dagger}$ Institute for Advanced Computer Studies, University of Maryland, College Park, MD 20742. 


\title{
On a Direct Method for the Solution of Nearly Uncoupled Markov Chains

\author{
G. W. StEWART* \\ G. ZHANG ${ }^{\dagger}$
}

\begin{abstract}
This note is concerned with the accuracy of the solution of nearly uncoupled Markov chains by a direct method based on the LU decomposition. It is shown that plain Gaussian elimination may fail in the presence of rounding errors. A modification of Gaussian elimination with diagonal pivoting and correction of small pivots is proposed and analyzed. It is shown that the accuracy of the solution is affected by two condition numbers associate with aggregation and the coupling respectively.
\end{abstract}

Abbreviated Title. Solution of NUMCs.

AMS(MOS) subject classification. Primary: 65F05, 65G05. Secondary: 15A51.

\section{Introduction}

In this note we will be concerned with the solution of a nearly uncoupled Markov chain (NUMC) whose transition matrix has the form

$$
\mathbf{P}^{\mathrm{T}}=\mathbf{D}+\mathbf{E}=\left(\begin{array}{cccc}
\mathbf{D}_{11} & \mathbf{E}_{12} & \cdots & \mathbf{E}_{1 k} \\
\mathbf{E}_{21} & \mathbf{D}_{22} & \cdots & \mathbf{E}_{2 k} \\
\vdots & \vdots & & \vdots \\
\mathbf{E}_{k 1} & \mathbf{E}_{k 2} & \cdots & \mathbf{D}_{t t}
\end{array}\right)
$$

where all the elements of the off-diagonal blocks $\mathbf{E}_{i j}$ are small. We will suppose that $\mathbf{P}$ is irreducible, so that $\mathbf{P}^{\mathrm{T}}$ has a unique positive eigenvector $\boldsymbol{\pi}$ corresponding to the eigenvalue one; i.e.,

$$
\mathbf{P}^{\mathrm{T}} \boldsymbol{\pi}=\boldsymbol{\pi} .
$$

${ }^{*}$ Department of Computer Science and Institute for Advanced Computer Studies, University of Maryland, College Park, MD 20742. This work was supported in part by the Air Force Office of Sponsored Research under Contract AFOSR-87-0188.

†Institute for Advanced Computer Studies, University of Maryland, College Park, MD 20742. 
We will normalize $\boldsymbol{\pi}$ so that the sum of its components is one; i.e.,

$$
\mathbf{1}^{\mathrm{T}} \boldsymbol{\pi}=1
$$

where

$$
\mathbf{1}^{\mathrm{T}}=(1,1, \cdots, 1) .
$$

Equivalently, if we set

$$
\mathbf{A}=\mathbf{I}-\mathbf{P}^{\mathrm{T}}
$$

we have

$$
\mathbf{A} \boldsymbol{\pi}=0 .
$$

Moreover, since $\mathbf{P}$ is stochastic, we have

$$
\mathbf{1}^{\mathrm{T}} \mathbf{A}=0 .
$$

In principle, the solution $\boldsymbol{\pi}$ can be obtained from the LU decomposition of A [3, 4]. Specifically, let $\mathbf{A}$ be transformed into an upper triangular matrix by Gaussian elimination; i.e., let

$$
\mathbf{M A}=\left(\begin{array}{cc}
\mathbf{U} & \mathbf{b} \\
\mathbf{0} & 0
\end{array}\right)
$$

where $\mathbf{U}$ is a nonsingular upper triangular matrix and $\mathbf{M}$ is lower triangular. The solution $\boldsymbol{\pi}$ can then be obtained by solving the equation

$$
\left(\begin{array}{ll}
\mathbf{U} & \mathbf{b} \\
\mathbf{0} & 0
\end{array}\right) \boldsymbol{\pi}=0
$$

subject to the normalization condition

$$
\mathbf{1}^{\mathrm{T}} \boldsymbol{\pi}=1 .
$$

Our chief concern is with the effect of rounding errors on this algorithm. We note that $\mathbf{A}$ is a singular M-matrix [2] and diagonally dominant [cf. (1.4)]. The results of the standard rounding-error analysis of Gaussian elimination show that the growth is bounded by one (see, e.g., [7, p.151], for the definition of the growth). Moreover, the backward errors have the same structure as $\mathbf{A}$, only they are smaller by a factor proportional to $\epsilon_{\mathrm{M}}$. At first glance this would seem to be an encouraging result. The perturbation theory for NUMCs shows that the solution $\boldsymbol{\pi}$ is 
insensitive to perturbations that are relatively small compared to $\mathbf{E}[10]$. However, the theory assumes that the sums of the columns of the perturbation are exactly equal to zero. When this condition is violated, even slightly, the solution $\boldsymbol{\pi}$ becomes quite sensitive to perturbations (see test problem 4 discuessed in [6]).

To see the failure of Gaussian elimination, we consider an example where the transition matrix is

$$
\mathbf{P}=\left(\begin{array}{ccc}
\frac{1}{2}-\epsilon & \frac{1}{2} & \epsilon \\
\frac{1}{2} & \frac{1}{2}-\epsilon & \epsilon \\
\epsilon & \epsilon & 1-2 \epsilon
\end{array}\right)
$$

Suppose $\epsilon$ is less than the machine precision. Then the matrix $\mathbf{A}$ is rounded to

$$
\mathbf{A}=\left(\begin{array}{rrr}
\frac{1}{2} & -\frac{1}{2} & -\epsilon \\
-\frac{1}{2} & \frac{1}{2} & -\epsilon \\
-\epsilon & -\epsilon & 0
\end{array}\right) .
$$

After the first column of $\mathbf{A}$ is annihilated by Gaussian elimination, the transformed matrix is rounded to

$$
\left(\begin{array}{rrr}
\frac{1}{2} & -\frac{1}{2} & -\epsilon \\
0 & 0 & -2 \epsilon \\
0 & -2 \epsilon & -2 \epsilon^{2}
\end{array}\right) .
$$

The process then breaks down owing to a zero pivot. Note also that the exact number at the last diagonal position should be $2 \epsilon-2 \epsilon^{2}$. A relative error of order unity is thus incurred in this diagonal element.

It should be stressed that the solution of the above problem is well determined and can be obtained through other methods, e.g., aggregation methods [8]. The failure is in Gaussian elimination itself.

Grassmann, Taksar and Heyman [5] have proposed a modification of Gaussian elimination which is claimed to give accurate solutions for NUMCs. The modification is based on the following observation. Let $\mathbf{A}$ be partitioned in the form

$$
\mathbf{A}=\left(\begin{array}{ll}
\mathbf{A}_{11} & \mathbf{A}_{12} \\
\mathbf{A}_{21} & \mathbf{A}_{22}
\end{array}\right),
$$

so that $\mathbf{A}_{11}$ is a nonsingular M-matrix. Let $\tilde{\mathbf{A}}_{22}$ be the Schur complement of $\mathbf{A}_{11}$; i.e., the result of performing Gaussian elimination through the block $\mathbf{A}_{11}$. Then $\tilde{\mathbf{A}}_{22}$ is a singular M-matrix and satisfies

$$
\mathbf{1}^{\mathrm{T}} \tilde{\mathbf{A}}_{22}=0 .
$$


This property enables us to correct the diagonal elements at each step of the elimination by replacing them with sums of the off-diagonal elements in the corresponding columns. It is easy to verify that this adjustment gives the correct solution in the above example. Unfortunately, we are unable to show whether the simple diagonal elements correction suggested in [5] will work for NUMCs with a large number of states.

In this note we are going to show that a related algorithm with diagonal pivoting will work for NUMCs with a large number of states. The norm $\|\cdot\|$ used in this note is the vector 2-norm and the subordinate matrix operator norm, respectively.

In Section 2 of this paper an algorithm for the solution of equation (1.3) is presented. A backward rounding-error analysis for the algorithm as well as a perturbation analysis are given in Section 3. Section 4 analyses the accuracy of the solution. Finally, some discussion appears in Section 5.

\section{Gaussian Elimination with Diagonal Adjustment}

In this section we present an algorithm for the solution $\boldsymbol{\pi}$ of a NUMC. Let $\mathbf{A}$ be partitioned in the form

$$
\mathbf{A}=\left(\begin{array}{cccc}
\mathbf{A}_{11} & -\mathbf{E}_{12} & \cdots & -\mathbf{E}_{1 t} \\
-\mathbf{E}_{21} & \mathbf{A}_{22} & \cdots & -\mathbf{E}_{2 t} \\
\vdots & \vdots & & \vdots \\
-\mathbf{E}_{t 1} & -\mathbf{E}_{t 2} & \cdots & \mathbf{A}_{t t}
\end{array}\right)
$$

where the diagonal block $\mathbf{A}_{k k}$ is an $m_{k} \times m_{k}$ matrix with $m_{k}$ being the number of states in the $k$ th aggregate. The total number of the states in the chain is

$$
n=m_{1}+m_{2}+\cdots+m_{t} .
$$

The off-diagonal blocks are assumed to satisfy

$$
\left\|\mathbf{E}_{i j}\right\| \leq \epsilon
$$

where $\epsilon$ is small. The following is an algorithm which combines Gaussian elimination with partial diagonal pivoting and diagonal correction.

\section{Algorithm.}


1. For $k=1,2, \cdots, t$,

1. Perform $m_{k}-1$ steps of Gaussian elimination on $\mathbf{A}$ with diagonal pivoting confined in the $k$ th diagonal block;

2. Bring the last pivot of the $k$ th diagonal block to the end of the current matrix and move successive rows and columns one step forward.

2. Transform the $t \times t$ matrix at the right-bottom corner to the upper triangular form by Gaussian elimination with each pivot being replaced by the sum of the off-diagonal elements of the pivot column.

3. Solve the resulting upper triangular system subject to the normalization condition (1.5).

4. Pre-multiply by the permutations of step 1 to bring the components of the solution to the right order.

In a language of matrix algebra, step 1 of this algorithm can be written as

$$
\mathbf{M}_{n-t} \mathbf{P}_{n-t} \mathbf{M}_{k-1} \cdots \mathbf{P}_{1} \mathbf{A} \mathbf{P}_{1}^{\mathrm{T}} \cdots \mathbf{P}_{n-t}^{\mathrm{T}}=\left(\begin{array}{cc}
\hat{\mathbf{U}}_{1} & \hat{\mathbf{B}} \\
\mathbf{0} & \hat{\mathbf{N}}+\hat{\mathbf{Z}}
\end{array}\right)
$$

where $\hat{\mathbf{U}}_{1}$ is an upper triangular matrix, $\hat{\mathbf{N}}$ is a diagonal matrix and $\hat{\mathbf{Z}}$ is an off-diagonal matrix, $\mathbf{P}_{i}$ is the permutation matrix which brings the last pivot of the $i$ th diagonal block to the end of the current matrix and $\mathbf{M}_{i}$ is the Gaussian transformation.

On the completion of step 2 is the upper triangular form

$$
\left(\begin{array}{cc}
\hat{\mathbf{U}} & \hat{\mathbf{b}} \\
\mathbf{0}^{\mathrm{T}} & 0
\end{array}\right)
$$

Step 3 and 4 amount to solving the equation

$$
\hat{\mathbf{U}} \mathbf{y}=-\hat{\mathbf{b}}
$$

and normalizing the solution $\boldsymbol{\pi}$ by

$$
\boldsymbol{\pi}=\mathbf{P}_{n-t} \cdots \mathbf{P}_{1}\left(\mathbf{y}^{\mathrm{T}}, 1\right)^{\mathrm{T}} /(\|\mathbf{y}\|+1)
$$


To analyse the accuracy of the solution computed by the algorithm, we set

$$
\hat{\mathbf{A}}=\mathbf{P}_{n-t} \cdots \mathbf{P}_{2} \mathbf{P}_{1} \mathbf{A} \mathbf{P}_{1}^{\mathrm{T}} \mathbf{P}_{2}^{\mathrm{T}} \cdots \mathbf{P}_{n-t}^{\mathrm{T}},
$$

and for $i=1,2, \cdots, n-t$

$$
\hat{\mathbf{M}}_{i}=\mathbf{P}_{n-t} \cdots \mathbf{P}_{i+1} \mathbf{M}_{i} \mathbf{P}_{i+1}^{\mathrm{T}} \cdots \mathbf{P}_{n-t}^{\mathrm{T}} \text {. }
$$

The operations of step 1 of the algorithm can then be written as

$$
\hat{\mathbf{M}}_{n-t} \cdots \hat{\mathbf{M}}_{1} \hat{\mathbf{A}}=\left(\begin{array}{cc}
\hat{\mathbf{U}}_{1} & \hat{\mathbf{B}} \\
\mathbf{0} & \hat{\mathbf{N}}+\hat{\mathbf{Z}}
\end{array}\right)
$$

That is, the process is equivalent to the elimination on $\hat{\mathbf{A}}$ by transformations $\hat{\mathbf{M}}_{1}, \cdots, \hat{\mathbf{M}}_{n-t}$ without diagonal pivoting. The matrix $\hat{\mathbf{A}}$ can be partitioned into the form

$$
\hat{\mathbf{A}}=\left(\begin{array}{ll}
\mathbf{K}+\mathbf{W} & \mathbf{L}+\mathbf{X} \\
\mathbf{M}+\mathbf{Y} & \mathbf{N}+\mathbf{Z}
\end{array}\right)
$$

where $\mathbf{K}, \mathbf{L}$ and $\mathbf{M}$ are block diagonal matrices consisting of $t$ blocks (not necessarily square) whose elements are of order unity, $\mathbf{N}$ is a $t \times t$ diagonal matrix of order unity and $\mathbf{W}, \mathbf{X}, \mathbf{Y}$ and $\mathbf{Z}$ are small off-diagonal matrices with their elements being of order $\epsilon$. The diagonal structures conform in the sense that all matrices have the same number of diagonal blocks and if the $i$ th block of $\mathbf{K}$ is $\left(m_{i}-1\right) \times\left(m_{i}-1\right)$ then the $i$ th blocks of $\mathbf{L}$ and $\mathbf{M}$ are $\left(m_{i}-1\right) \times 1$ and $1 \times\left(m_{i}-1\right)$ respectively.

The Schur complement of $\mathbf{K}+\mathbf{W}$ in $\hat{\mathbf{A}}$ is

$$
\hat{\mathbf{N}}+\hat{\mathbf{Z}}=\mathbf{N}+\mathbf{Z}-(\mathbf{M}+\mathbf{Y})(\mathbf{K}+\mathbf{W})^{-1}(\mathbf{L}+\mathbf{X}) \text {. }
$$

In the above algorithm it is this Schur complement whose diagonal elements are corrected by the sums of the off-diagonal elements in the corresponding columns. In the next section we show that the off-diagonal part $\hat{\mathbf{Z}}$ can be accurately obtained in the presence of rounding errors.

\section{The Accuracy of $\hat{Z}$}

In this section we consider the accuracy of the off-diagonal part $\hat{\mathbf{Z}}$ of the Schur complement (2.3) obtained by executing step 1 of the algorithm in the presence of 
rounding errors. Since $\hat{\mathbf{A}}$ is obtained from $\mathbf{A}$ only through digaonal permutations, $\hat{\mathbf{A}}$ is a singular M-matrix satisfying

$$
\mathbf{1}^{\mathrm{T}} \hat{\mathbf{A}}=0 .
$$

The property (3.1) is preserved in the submatrices generated through Gaussian elimination.

To look at th rounding errors involved in Step 1 of the algorithm, we rewrite the matrix $\hat{\mathbf{A}}$ in element-wise notation; i.e., let

$$
\hat{\mathbf{A}}=\left(\alpha_{i j}\right) \text {. }
$$

Following the analysis in $[7, \mathrm{p} .410-411]$ and noticing that

$$
\begin{aligned}
\left|\alpha_{i j}^{(k+1)}\right| & =\left|\alpha_{i j}^{(k)}-\frac{\alpha_{i k}^{(k)}}{\alpha_{k k}^{(k)}} \alpha_{k j}^{(k)}\right| \\
& =\left|\alpha_{i j}^{(k)}\right|+\left|\frac{\alpha_{i k}^{(k)}}{\alpha_{k k}^{(k)}} \alpha_{k j}^{(k)}\right|,
\end{aligned}
$$

we have that the backward rounding errors introduced in the $(i, j)$-elements for $i \neq j$ by the elimination are of the form

$$
c \cdot \epsilon_{\mathrm{M}} \max _{k}\left\{\left|\alpha_{i j}^{(k)}\right|\right\},
$$

where $\epsilon_{\mathrm{M}}$ is the rounding unit for the computer, $c$ is a constant of at most order $n$ and $\alpha_{i j}^{(k)}$ is the $(i, j)$-element on the completion of $k-1$ steps of elimination.

Since the diagonal elements are bounded by one, the rounding errors at the diagonal positions are of order $\epsilon_{\mathrm{M}}$. To bound the rounding errors involved at offdiagonal positions, we only need to consider the growth of the upper off-diagonal elements in the elimination process. Since the absolute value of $\alpha_{i j}^{(k)}$ increases monotonically with $k$, we have

$$
\max _{k}\left\{\left|\alpha_{i j}^{(k)}\right|\right\}=\left|\alpha_{i j}^{(i)}\right|, \quad i<j .
$$

Theorem 3.1. The growth of upper off-diagonal elements are bounded by the following inequality

$$
\left|\alpha_{i j}^{(i)}\right| \leq\left|\alpha_{i j}\right|+\sqrt{i-1} \kappa_{i} \sum_{s=1}^{i-1}\left|\alpha_{s j}\right|, \quad i<j,
$$

where $\kappa_{i}$ is the 2-norm of the inverse of the $(i-1)$ th leading principal matrix of $\hat{\mathbf{A}}$ and $\kappa_{1}=0$. 
Proof. The $i$ th row of the final upper triangular matrix is the result of eliminating the row vector $\left(\alpha_{i 1} \alpha_{i 2} \cdots \alpha_{i i-1}\right)$ by pre-multiplying $\hat{\mathbf{A}}$ the block matrix

$$
\left(\begin{array}{ccc}
\mathbf{I} & \mathbf{0} & \mathbf{0} \\
\mathbf{m}_{i}^{\mathrm{T}} & 1 & \mathbf{0} \\
\mathbf{0} & \mathbf{0} & \mathbf{I}
\end{array}\right)
$$

where

$$
\mathbf{m}_{i}^{\mathrm{T}}=-\left(\begin{array}{llll}
\alpha_{i 1} & \alpha_{i 2} & \cdots & \alpha_{i, i-1}
\end{array}\right)\left(\begin{array}{cccc}
\alpha_{11} & \alpha_{12} & \cdots & \alpha_{1, i-1} \\
\alpha_{21} & \alpha_{22} & \cdots & \alpha_{2, i-1} \\
\vdots & \vdots & & \vdots \\
\alpha_{i-1,1} & \alpha_{i-1,2} & \cdots & \alpha_{i-1, i-1}
\end{array}\right)^{-1}
$$

Therefore

$$
\alpha_{i j}^{(i)}=\alpha_{i j}+\mathbf{m}_{i}^{\mathrm{T}}\left(\begin{array}{c}
\alpha_{1 j} \\
\vdots \\
\alpha_{i-1, j}
\end{array}\right) .
$$

Since $\left|\alpha_{s t}\right|$ are less than one, the conclusion follows by taking the norm of the second term of the right hand side of the above equality.

Since the diagonal pivoting has been performed and the last pivot of each block (which is of order $\epsilon$ ) has been permuted to the end of the current matrix in the elimination process, it is reasonable to assume that the successive leading principal submatrices are well-conditioned; i.e., $\kappa_{i}$ are all of order unity. If $\alpha_{i j}$ is of order one, $\alpha_{1 j}$ through $\alpha_{i-1, j}$ are all of order one. If $\alpha_{i j}$ is of order $\epsilon, \alpha_{1 j}$ through $\alpha_{i-1, j}$ are all of order $\epsilon$. This follows that $\alpha_{i j}^{(i)}$ is of the same order as that of $\alpha_{i j}$.

From the above analysis, we know there is a matrix $\mathbf{E}_{\mathrm{M}}$ such that

$$
\hat{\mathbf{M}}_{k} \cdots \hat{\mathbf{M}}_{1}\left(\hat{\mathbf{A}}+\mathbf{E}_{\mathrm{M}}\right)=\left(\begin{array}{cc}
\overline{\mathbf{U}}_{1} & \overline{\mathbf{B}} \\
\mathbf{0} & \overline{\mathbf{N}}+\overline{\mathbf{Z}}
\end{array}\right) .
$$

Here we have used the upper bar to denote the computed values of matrices $\hat{\mathbf{U}}_{1}$, $\hat{\mathbf{B}}$, etc. The important feature of $\mathbf{E}_{\mathrm{M}}$ is that it has the same block structure as that of matrix $\mathbf{A}$; namely, the block of $\mathbf{E}_{\mathrm{M}}$ corresponding to the block of order 
unity in $\hat{\mathbf{A}}$ is of order $\epsilon_{\mathrm{M}}$ while the block of $\mathbf{E}_{\mathrm{M}}$ corresponding to the block of order $\epsilon$ in $\hat{\mathbf{A}}$ is of order $\epsilon \epsilon_{\mathrm{M}}$.

The next step is to bound the difference between $\overline{\mathbf{Z}}$ and $\hat{\mathbf{Z}}$. For most transition matrices of NUMCs, it is reasonable to expect that $\mathbf{K}, \mathbf{L}, \mathbf{M}$ and $\mathbf{N}$ are all approximately the same size. Hence we assume that

$$
\|\mathbf{K}\|=\|\mathbf{L}\|=\|\mathbf{M}\|=\|\mathbf{N}\|=\alpha .
$$

The matrices $\mathbf{W}, \mathbf{X}, \mathbf{Y}$ and $\mathbf{Z}$ are small compared to $\alpha$; that is

$$
\|\mathbf{W}\|=\|\mathbf{X}\|=\|\mathbf{Y}\|=\|\mathbf{Z}\| \leq \alpha \epsilon .
$$

The matrix $\mathbf{K}$ is nonsingular, and we set

$$
\eta=\left\|\mathbf{K}^{-1}\right\| \text { and } \kappa=\alpha \eta .
$$

Let $\tilde{\mathbf{K}}, \tilde{\mathbf{W}}, \tilde{\mathbf{L}}$, etc. be perturbations of $\mathbf{K}, \mathbf{W}$, $\mathbf{L}$, etc., then from the above backward rounding-error analysis we can assume that

$$
\|\tilde{\mathbf{K}}-\mathbf{K}\|=\|\tilde{\mathbf{L}}-\mathbf{L}\|=\|\tilde{\mathbf{M}}-\mathbf{M}\|=\|\tilde{\mathbf{N}}-\mathbf{N}\| \leq \alpha \epsilon_{\mathrm{M}}
$$

and

$$
\|\tilde{\mathbf{W}}-\mathbf{W}\|=\|\tilde{\mathbf{X}}-\mathbf{X}\|=\|\tilde{\mathbf{Y}}-\mathbf{Y}\|=\|\tilde{\mathbf{Z}}-\mathbf{Z}\| \leq \alpha \epsilon \epsilon_{\mathrm{M}} .
$$

Since $\epsilon$ and $\epsilon_{\mathrm{M}}$ are far less than 1, we may ignore terms of order $\epsilon \epsilon_{\mathrm{M}}^{2}$ and $\epsilon^{2} \epsilon_{\mathrm{M}}$. In the proof of the following theorem $\Delta(\mathbf{T})$ is a generic bound for the perturbation of $\mathbf{T}$ that is accurate up to terms of order $\epsilon \epsilon_{\mathrm{M}}^{2}$ and $\epsilon^{2} \epsilon_{\mathrm{M}}$.

\section{Theorem 3.2.}

$$
\Delta(\hat{\mathbf{Z}})=\left(1+4 \kappa+5 \kappa^{2}+2 \kappa^{3}\right) \alpha \epsilon \epsilon_{\mathrm{M}} .
$$

Proof. We begin by writing $(\mathbf{K}+\mathbf{W})^{-1}=\mathbf{K}^{-1}-\mathbf{K}^{-1} \mathbf{W} \mathbf{K}^{-1}+O\left(\epsilon^{2}\right)$. It follows that

$$
\hat{\mathbf{N}}+\hat{\mathbf{T}}=\mathbf{T}-(\mathbf{M}+\mathbf{Y}) \mathbf{K}^{-1}(\mathbf{L}+\mathbf{X})+\mathbf{M} \mathbf{K}^{-1} \mathbf{W} \mathbf{K}^{-1} \mathbf{L}+O\left(\epsilon^{2}\right) .
$$

Now terms like $\mathbf{M} \mathbf{K}^{-1} \mathbf{L}$ are diagonal, while terms like $\mathbf{M} \mathbf{K}^{-1} \mathbf{X}$ are off-diagonal. It follows that

$$
\hat{\mathbf{Z}}=\mathbf{Z}-\mathbf{M} \mathbf{K}^{-1} \mathbf{X}-\mathbf{Y} \mathbf{K}^{-1} \mathbf{L}+\mathbf{M K}^{-1} \mathbf{W} \mathbf{K}^{-1} \mathbf{L}+O\left(\epsilon^{2}\right) .
$$

The rest of the proof consists of a straightforward, if tedious evaluation of $\Delta$ for each of the terms in (3.4). Here we make free use of the fact that

$$
\Delta(\mathbf{R S T})=\Delta(\mathbf{R})\|\mathbf{S} \mid\| \mathbf{T}\|+\| \mathbf{R}\|\Delta(\mathbf{S})\| \mathbf{T}\|+\| \mathbf{R}\|\| \mathbf{S} \| \Delta(\mathbf{T}) .
$$


1. $\Delta(\mathbf{Z})=\alpha \epsilon \epsilon_{\mathrm{M}}$

2. $\Delta\left(\mathbf{K}^{-1}\right)=\alpha \eta^{2} \epsilon_{\mathrm{M}}$

3. $\Delta\left(\mathbf{Y K}^{-1} \mathbf{L}\right)=\left(\alpha \epsilon_{\mathrm{M}} \epsilon\right)(\eta)(\alpha)+(\alpha \epsilon)\left(\alpha \eta^{2} \epsilon_{\mathrm{M}}\right)(\alpha)+(\alpha \epsilon)(\eta)\left(\alpha \epsilon_{\mathrm{M}}\right)$ $=\left(2 \kappa+\kappa^{2}\right) \alpha \epsilon \epsilon_{\mathrm{M}}$

4. $\Delta\left(\mathbf{M K}^{-1} \mathbf{X}\right)=\left(2 \kappa+\kappa^{2}\right) \alpha \epsilon \epsilon_{\mathrm{M}}$

5. $\Delta\left(\mathbf{M K}^{-1}\right)=\left(\alpha \epsilon_{\mathrm{M}}\right)(\eta)+(\alpha)\left(\alpha \eta^{2} \epsilon_{\mathrm{M}}\right)$ $=\left(\kappa+\kappa^{2}\right) \epsilon_{\mathrm{M}}$

6. $\Delta\left(\mathbf{K}^{-1} \mathbf{L}\right)=\left(\kappa+\kappa^{2}\right) \epsilon_{\mathrm{M}}$

7. $\Delta\left(\mathbf{M K}^{-1} \mathbf{W K}^{-1} \mathbf{L}\right)=2\left[\left(\kappa+\kappa^{2}\right) \epsilon_{\mathrm{M}}\right](\alpha \epsilon)(\kappa)+(\kappa)\left(\alpha \epsilon \epsilon_{\mathrm{M}}\right)(\kappa)$

$$
=\left(3 \kappa^{2}+2 \kappa^{3}\right) \alpha \epsilon \epsilon_{\mathrm{M}}
$$

The bound (3.3) now follows on adding items 1, 3, 4, and 7 in the above list.

The theorem (3.2) shows that off-diagonal elements of the Schur complement are accurately obtained with relative error of order $\epsilon_{M}$ if $\kappa$ is not very large. The diagonal correction in step 2 of the algorithm brings the accuracy back to the pivots which may otherwise have high relative error due to cancellation.

\section{The Accuracy of the Solution}

Let

$$
\hat{\boldsymbol{\pi}}=\mathbf{P}_{1}^{\mathrm{T}} \cdots \mathbf{P}_{n-t}^{\mathrm{T}} \boldsymbol{\pi}=\left(\hat{\boldsymbol{\pi}}_{1}^{\mathrm{T}}, \hat{\boldsymbol{\pi}}_{2}^{\mathrm{T}}\right)^{\mathrm{T}}
$$

be the partition conforming with (2.2). Then

$$
\left(\begin{array}{cc}
\mathbf{K}+\mathbf{W} & \mathbf{L}+\mathbf{X} \\
\mathbf{0} & \hat{\mathbf{N}}+\hat{\mathbf{Z}}
\end{array}\right)\left(\begin{array}{c}
\hat{\boldsymbol{\pi}}_{1} \\
\hat{\boldsymbol{\pi}}_{2}
\end{array}\right)=0
$$

i.e., the solution is obtained by solving

$$
(\hat{\mathbf{N}}+\hat{\mathbf{Z}}) \hat{\boldsymbol{\pi}}_{2}=0 .
$$

and

$$
(\mathbf{K}+\mathbf{W}) \hat{\boldsymbol{\pi}}_{1}=-(\mathbf{L}+\mathbf{X}) \hat{\boldsymbol{\pi}}_{2}
$$


The matrix $\hat{\mathbf{N}}+\hat{\mathbf{Z}}$ is a singular M-matrix of order $\epsilon$ and the sums of its columns are zero; i.e.,

$$
\mathbf{1}^{\mathrm{T}}(\hat{\mathbf{N}}+\hat{\mathbf{Z}})=0
$$

In the presence of rounding errors, the coefficient matrix of (4.3) is replaced by $\overline{\mathbf{N}}+\overline{\mathbf{Z}}$. The analysis of the last section shows that $\|\overline{\mathbf{Z}}-\hat{\mathbf{Z}}\|$ is of order $\epsilon \epsilon_{\mathrm{M}}$. Notice in the step 2 of the algorithm that Gaussian elimination is performed with each pivot being repalced by the sum of the column of $\overline{\mathbf{Z}}$. The rounding errors thus introduced are at most of order $\epsilon \epsilon_{\mathrm{M}}$. Moreover, the final upper triangular has positive diagonals (except the last one which is set to zero) and nonpositive off-diagonals. The solution of such a system is computed to high accuracy [11, p.249-251]. Therefore, we can assume that the computed vector $\overline{\boldsymbol{\pi}}_{2}$ of $\hat{\boldsymbol{\pi}}_{2}$ satisfies

$$
[(\hat{\mathbf{N}}+\delta \hat{\mathbf{N}})+(\hat{\mathbf{Z}}+\delta \hat{\mathbf{Z}})] \overline{\boldsymbol{\pi}}_{2}=0
$$

where $\delta \hat{\mathbf{N}}$ and $\delta \hat{\mathbf{Z}}$ are matrices of order $\epsilon \epsilon_{\mathrm{M}}$.

It has been proved in [1] that

$$
\begin{aligned}
\frac{\left\|\overline{\boldsymbol{\pi}}_{2}-\hat{\boldsymbol{\pi}}_{2}\right\|}{\left\|\overline{\boldsymbol{\pi}}_{2}\right\|} & \leq \frac{(1+\sqrt{t})\|\delta \hat{\mathbf{N}}+\delta \hat{\mathbf{Z}}\|}{\sigma_{t-1}} \\
& \leq C \frac{\epsilon \epsilon_{\mathrm{M}}}{\sigma_{t-1}}
\end{aligned}
$$

where $\sigma_{t-1}$ is the smallest positive singular value of $\hat{\mathbf{N}}+\hat{\mathbf{Z}}$ and $C$ is a constant independent with $\epsilon$. We will call $\sigma_{t-1}^{-1}$ the condition number with respect to coupling.

The accuracy of $\hat{\boldsymbol{\pi}}_{1}$ relies on the accuracy of $\hat{\boldsymbol{\pi}}_{2}$ as well as the condition of $\mathbf{K}$. The analysis of last section shows that the computed vector $\overline{\boldsymbol{\pi}}_{1}$ of $\hat{\boldsymbol{\pi}}_{1}$ satisfies

$$
(\tilde{\mathbf{K}}+\hat{\mathbf{W}}) \overline{\boldsymbol{\pi}}_{1}=-(\tilde{\mathbf{L}}+\tilde{\mathbf{X}}) \overline{\boldsymbol{\pi}}_{2}
$$

Note that the rounding errors introduced in the back substitution have been neglected in the above equality because the solution of the corresponding upper triangular system is computed to high accuracy by the fact of the favorable signs of elements in the upper triangular and the right hand side [11, p.249-251]. By the standard first order bounds of $(\mathbf{K}+\mathbf{W})^{-1}$, the error in $\hat{\boldsymbol{\pi}}_{1}$ is bounded by the following inequality

$$
\left\|\overline{\boldsymbol{\pi}}_{1}-\hat{\boldsymbol{\pi}}_{1}\right\| \leq \kappa \epsilon_{\mathrm{M}}\left\|\overline{\boldsymbol{\pi}}_{1}\right\|+C\left(1+\frac{\epsilon}{\sigma_{t-1}}\right) \kappa \epsilon_{\mathrm{M}}\left\|\overline{\boldsymbol{\pi}}_{2}\right\|+o\left(\epsilon_{\mathrm{M}}\right)
$$


where $\kappa$ is defined by (3.2). We call $\kappa$ the condition number with respect to aggregation. For UNMCs with two condition numbers being

$$
\sigma_{t-1}^{-1}=O\left(\epsilon^{-1}\right) \text { and } \kappa=O(1)
$$

the solution obtained by the algorithm satisfies

$$
\Delta(\boldsymbol{\pi})=O\left(\epsilon_{\mathrm{M}}\right)
$$

no matter how small $\epsilon$ is.

\section{Discussion}

It has been shown that the accuracy of the solution depends on two numbers $\sigma_{t-1}^{-1}$ and $\kappa$. The relation of these two numbers with the NUMC can be illustrated by considering a NUMC with two aggregates. To this end, we write the block structure of $\mathbf{K}, \mathbf{L}, \mathbf{W}$ and $\mathbf{X}$ explicitly as follows

$$
\begin{gathered}
\mathbf{K}=\left(\begin{array}{cc}
\mathbf{K}_{1} & \mathbf{0} \\
\mathbf{0} & \mathbf{K}_{2}
\end{array}\right), \quad \mathbf{L}=\left(\begin{array}{cc}
\mathbf{l}_{1} & \mathbf{0} \\
\mathbf{0} & \mathbf{l}_{2}
\end{array}\right), \\
\mathbf{W}=\left(\begin{array}{cc}
\mathbf{0} & \mathbf{W}_{2} \\
\mathbf{W}_{1} & \mathbf{0}
\end{array}\right), \quad \mathbf{X}=\left(\begin{array}{cc}
\mathbf{0} & \mathbf{x}_{2} \\
\mathbf{x}_{1} & \mathbf{0}
\end{array}\right),
\end{gathered}
$$

where $\mathbf{K}_{i}$ and $\mathbf{l}_{i}$ are of order unity, $\mathbf{W}_{i}$ and $\mathbf{x}_{i}$ are of order $\epsilon$. The solution $\hat{\boldsymbol{\pi}}$ of (4.1) is partitioned conformaly as

$$
\hat{\boldsymbol{\pi}}_{1}=\left(\hat{\boldsymbol{\pi}}_{11}^{\mathrm{T}}, \hat{\boldsymbol{\pi}}_{21}^{\mathrm{T}}\right)^{\mathrm{T}}, \quad \hat{\boldsymbol{\pi}}_{2}=\left(\hat{\pi}_{12}, \hat{\pi}_{22}\right)^{\mathrm{T}} .
$$

A partition of the solution with respect to the aggregates is thus given by

$$
\begin{aligned}
& \left(\begin{array}{l}
\hat{\boldsymbol{\pi}}_{11} \\
\hat{\pi}_{12}
\end{array}\right) \text { the portion of the solution w.r.t. aggregate } 1, \\
& \left(\begin{array}{c}
\hat{\boldsymbol{\pi}}_{21} \\
\hat{\pi}_{22}
\end{array}\right) \text { the portion of the solution w.r.t. aggregate } 2 .
\end{aligned}
$$


By the first order expansion of the inverse of $(\mathbf{K}+\mathbf{W})$ and from equation (4.2), we have

$$
\begin{aligned}
\left(\begin{array}{l}
\hat{\boldsymbol{\pi}}_{11} \\
\hat{\boldsymbol{\pi}}_{21}
\end{array}\right)= & \left(\begin{array}{cc}
\mathbf{K}_{1}^{-1} & \mathbf{0} \\
\mathbf{0} & \mathbf{K}_{2}^{-1}
\end{array}\right)\left(\begin{array}{cc}
\mathbf{l}_{1} & \mathbf{0} \\
\mathbf{0} & \mathbf{l}_{2}
\end{array}\right)\left(\begin{array}{l}
\hat{\pi}_{12} \\
\hat{\pi}_{22}
\end{array}\right)+ \\
& \left(\begin{array}{cc}
\mathbf{K}_{1}^{-1} & \mathbf{0} \\
\mathbf{0} & \mathbf{K}_{2}^{-1}
\end{array}\right)\left(\begin{array}{cc}
\mathbf{0} & \mathbf{x}_{2} \\
\mathbf{x}_{1} & \mathbf{0}
\end{array}\right)\left(\begin{array}{l}
\hat{\pi}_{12} \\
\hat{\pi}_{22}
\end{array}\right)+O\left(\epsilon^{2}\right)
\end{aligned}
$$

The important fact can be read from equality (5.1) is that the approximate solution accurate to the order of $\epsilon$ corresponding to the aggregates is

$$
\hat{\pi}_{12}\left(\begin{array}{c}
\mathbf{K}_{1}^{-1} \mathbf{l}_{1} \\
1
\end{array}\right),
$$

and

$$
\hat{\pi}_{22}\left(\begin{array}{c}
\mathbf{K}_{2}^{-1} \mathbf{l}_{2} \\
1
\end{array}\right) .
$$

Except the scaling factors $\hat{\pi}_{12}$ and $\hat{\pi}_{22}$, these approximations are determined by the matrices of order unity associate with their corresponding aggregates. When all the matrices $\mathbf{K}_{i}$ are well-conditioned; i.e., $\kappa$ is small, they are insensitive to the variation of $\epsilon$. Therefore, it is legitimate to define $\kappa$ as the condition number corresponding to aggregates.

The scaling factors $\hat{\pi}_{12}$ and $\hat{\pi}_{2}$ are the solution of the singular system (4.3). As we have seen before, the coefficient matrix $\hat{\mathbf{N}}+\hat{\mathbf{Z}}$ is of order $\epsilon$. This indicates that $\hat{\pi}_{12}$ and $\hat{\pi}_{22}$ will reflect the effect of coupling between aggregates. We will call these scaling factors the coupling coefficients. When the condition number $\sigma_{t-1}^{-1}$ is not too large compared to $\epsilon^{-1}$, these coupling coefficients are insensitive to the perturbations which are relatively small compared to $\epsilon$.

It should be noted that these two condition numbers are related to the regularity conditions in [9], [10]. A small $\kappa$ means that there is only one eigenvalue of $\mathbf{D}_{i i}$ approaching one as $\epsilon \rightarrow 0$ while the rest of the eigenvalues remain away from the unity. This is implied in the second regularity condition in [9], [10]. The relation between the first regularity condition and $\sigma_{t-1}$ can be seen from the following observation. Suppose that $\sigma_{t-1}$ is much smaller than $\epsilon$. By using the fact that $\hat{\mathbf{N}}+\hat{\mathbf{Z}}$ is a singular M-matrix, it is easy to verify that at least one components of the solution $\hat{\boldsymbol{\pi}}_{2}$ of equation (4.3) is of order $\sigma_{t-1} \epsilon^{-1}$ when $\hat{\boldsymbol{\pi}}_{2}$ is normalized to have $\left\|\hat{\boldsymbol{\pi}}_{2}\right\|=1$. In other words, at least one of the coupling coeifficients is very 
small. In this case the first regularity condition is violated. Therefore, the first regularity condition requires that $\sigma_{t-1}$ should not be too small compared to $\epsilon$.

The analysis of this paper leaves open the question about whether the simple diagonal adjustment without diagonal pivoting works for NUMCs with large state number.

\section{References}

[1] J. L. Barlow (1986). "On the Smallest Positive Singular Value of a Singular M-Matrix with Applications to Ergodic Markov Chains." SIAM J. Alg. Disc. Meth., 7, 414-424.

[2] A. Berman and R. Plemmons (1979). Nonnegative Matrices in the Mathematical Sciences. Academic Press, New York.

[3] R. E. Funderlic and J.B. Mankin (1981). "Solution of homogeneous systems of equations arising from compartmental models." SIAM J. Sci. Statist. Comput., 2, 375-383.

[4] R. E. Funderlic and R.J. Plemmons (1981). "LU decomposition of M-matrices by elimination without pivoting." Linear Algebra and Its Applications, 44, $99-110$.

[5] W. K. Grassmann, M. I. Taksar, and D. P. Heyman (1985). "Regenerative Analysis and Steady State Distributions for Markov Chains." Operations Research, 33, 1107-1116.

[6] W. J. Harrod and R. J. Plemmons (1984). "Comparisons of Some Direct Methods for Computing Stationary Distributions of Markov Chains." SIAM J. Sci Statist. Comput., 5, 453-469.

[7] G. W. Stewart (1973). Introduction to Matrix Computations. Academic Press, New York.

[8] G. W. Stewart (1983). "Computable Error Bounds for Aggregated Markov Chains." Journal of the Association for Computing Machinery, 30, 271-285.

[9] G. W. Stewart (1984). "On the Structure of Nearly Uncoupled Markov Chains." In G. Iazeolla, P. J. Courtois, and A. Hordijk, editors, Mathematical Computer Performance and Reliability, pages 287-302, North Holland. Elsevier. 
[10] G. W. Stewart (1990). "On the Sensitivity of Nearly Uncoupled Markov Chains." Technical Report CS-TR 2406, Department of Computer Science, University of Maryland.

[11] J. H. Wilkinson (1965). The Algebraic Eigenvalue Problem. Clarendon Press, Oxford. 